\title{
Dedekind sums and continued fractions
}

\author{
by
}

R. R. Hall (York) and M. N. Huxley (Cardiff)

Let $\varrho(t)$ denote the row-of-teeth function

$$
\varrho(t)=[t]-t+1 / 2 .
$$

Let $a, b, c, \ldots, p, q, r$ be positive integers. The homogeneous Dedekind sum is

$$
D(a, b ; q)=\sum_{t=1}^{q-1} \varrho\left(\frac{a t}{q}\right) \varrho\left(\frac{b t}{q}\right),
$$

and the inhomogeneous Dedekind sum is

$$
D\left(\frac{p}{q}\right)=\sum_{t=1}^{q-1} \varrho\left(\frac{t}{q}\right) \varrho\left(\frac{p t}{q}\right)=D(1, p ; q) .
$$

The homogeneous sum can be expressed in terms of inhomogeneous sums using the relation

$$
\sum_{u=0}^{q-1} \varrho\left(t+\frac{u}{q}\right)=\varrho(q t)
$$

to obtain the following rules:

$$
\begin{gathered}
D(a c, b c ; c q)=c D(a, b ; q)+(c-1) / 4 \quad \text { for any } c, \\
D(a c, b c ; q)=D(a, b ; q) \quad \text { for }(c, q)=1, \\
D(a, b c ; c q)=D(a, b ; q) \quad \text { for }(a, c)=1 .
\end{gathered}
$$

Putting $a=1$ in (4), we find that the inhomogeneous sum is a well-defined function of a positive rational argument. Since $D(a, b ; q)$ depends only on $a$ and $b$ modulo $q$, we can reduce the homogeneous sum $D(a, b ; q)$ to the inhomogeneous sum in two different ways, either by choosing $c$ so that $a c \equiv 1$ $(\bmod q)$, or so that $b c \equiv 1(\bmod q)$. We thus find that

$$
D(p / q)=D(r / q) \quad \text { when } p r \equiv 1(\bmod q) .
$$


A final trivial relation

$$
D(p / q)=-D((b q-p) / q),
$$

for any large integer $b$, follows from the oddness of the function $\varrho(t)$.

In this note we base the theory of the inhomogeneous Dedekind sum on the continued fraction algorithm. Our main tool is a three-term recurrence relation for $D(p / q)$, which gives a direct proof of the reciprocity formula

$$
D\left(\frac{p}{q}\right)+D\left(\frac{q}{p}\right)=\frac{p^{2}+q^{2}+1}{12 p q}-\frac{1}{4},
$$

and Barkan and Hickerson's evaluation of $D(p / q)[1,5]$ (which is also implicit in Knuth [6]). The recurrence relation extends to give an interpolation formula for inhomogeneous Dedekind sums, our Theorem 2 below, which appears to be a new result.

The reciprocity formula (7) is a special case of the functional equation, valid for $p, q, r$ positive and pairwise coprime,

$$
D(p, q ; r)+D(q, r ; p)+D(r, p ; q)=\frac{p^{2}+q^{2}+r^{2}}{12 p q r}-\frac{1}{4} .
$$

It appears that (8) cannot be deduced directly from the special case (7). A simple proof of (8) using integration will be given elsewhere. Other proofs of the functional equation (8) use contour integration, or trigonometric identities among roots of unity, or counting the lattice points in a certain tetrahedron.

The association of $D(p / q)$ with continued fractions is natural, since the Dedekind sums first arose in connection with the multiplier system for Dedekind's eta function over the modular group of two by two integer matrices of determinant one [4]. The continued fraction algorithm can be interpreted as the expression of a matrix of the modular group as a word in the generators $\left(\begin{array}{ll}1 & 1 \\ 0 & 1\end{array}\right)$ and $\left(\begin{array}{ll}1 & 0 \\ 1 & 1\end{array}\right)$.

The continued fraction for $p / q$ is calculated using Euclid's algorithm. Let $r_{0}=q$, and let

$$
p=a_{0} r_{0}+r_{1}, \quad 0 \leq r_{1} \leq q-1, a_{0} \geq 0 .
$$

If $r_{1} \neq 0$, then let

$$
r_{0}=a_{1} r_{1}+r_{2}, \quad 0 \leq r_{2} \leq r_{1}-1, a_{1} \geq 1,
$$

and so on. Eventually $r_{n+1}=0$ for some $n \geq 0$. The convergents are

$$
p_{i} / q_{i}=a_{0}+1 /\left(a_{1}+1 /\left(a_{2}+\ldots+1 / a_{i}\right) \ldots\right),
$$

satisfying the three-term recurrences

$$
p_{i+1}=a_{i+1} p_{i}+p_{i-1}, \quad q_{i+1}=a_{i+1} q_{i}+q_{i-1},
$$


with

$$
\frac{p_{i+1}}{q_{i+1}}-\frac{p_{i}}{q_{i}}=\frac{(-1)^{i}}{q_{i} q_{i+1}} .
$$

The last convergent $p_{n} / q_{n}$ is the lowest terms reduction of the ratio $p / q$.

TheOREM 1 (Barkan's evaluation [1]). Let $n$ be the length of the continued fraction for $p / q$. Then

$$
D\left(\frac{p}{q}\right)= \begin{cases}\frac{1}{12}\left(\frac{p_{n}-q_{n-1}}{q_{n}}-a_{0}+a_{1}-\ldots-a_{n}\right) & \text { for } n \text { even } \\ \frac{1}{12}\left(\frac{p_{n}+q_{n-1}}{q_{n}}-a_{0}+a_{1}-\ldots+a_{n}\right)-\frac{1}{4} & \text { for } n \text { odd } .\end{cases}
$$

Since

$$
q_{n-1} / q_{n}=1 /\left(a_{n}+1 /\left(a_{n-1}+\ldots\left(a_{2}+1 / a_{1}\right) \ldots\right)\right),
$$

the expressions in the theorem are symmetric in $p_{n}$ and $q_{n-1}$, in accordance with (5) and (9).

Our evaluation of the Dedekind sum rests on the three-term recurrence that follows.

Lemma 1. Let $a, p, p^{\prime}, p^{\prime \prime}, q, q^{\prime}, q^{\prime \prime}$ be positive integers with

$$
\begin{gathered}
p=a p^{\prime}+p^{\prime \prime}, \quad q=a q^{\prime}+q^{\prime \prime}, \\
\frac{p}{q}-\frac{p^{\prime}}{q^{\prime}}= \pm \frac{1}{q q^{\prime}} .
\end{gathered}
$$

Then

$$
\begin{aligned}
D\left(\frac{p}{q}\right)=\left(1-\frac{q^{\prime \prime}}{q}\right) D\left(\frac{p^{\prime}}{q^{\prime}}\right)+ & \frac{q^{\prime \prime}}{q} D\left(\frac{p^{\prime \prime}}{q^{\prime \prime}}\right) \\
& \pm\left(\frac{a}{12}\left(1+\frac{q^{\prime \prime}}{q}\right)-\frac{1}{4}\left(1-\frac{q^{\prime \prime}}{q}\right)\right),
\end{aligned}
$$

the \pm sign in (12) agreeing with that in (11).

P r o of. If the sign in (11) is negative, then we replace $p, p^{\prime}, p^{\prime \prime}$ by $b q-p$, $b q^{\prime}-p^{\prime}, b q^{\prime \prime}-p^{\prime \prime}$ for some large integer $b$; the sign becomes positive, and the Dedekind sums have changed sign by (6). Hence we may assume a positive sign in (11).

We observe that for $0 \leq t<q$ we have

$$
\varrho\left(\frac{p t}{q}\right)=\varrho\left(\frac{p^{\prime} t}{q^{\prime}}+\frac{t}{q q^{\prime}}\right)=\varrho\left(\frac{p^{\prime} t}{q^{\prime}}\right)-\frac{t}{q q^{\prime}},
$$

and similarly for $1 \leq t<q^{\prime}, t$ not a multiple of $q^{\prime \prime}$, we have

$$
\varrho\left(\frac{p^{\prime} t}{q^{\prime}}\right)=\varrho\left(\frac{p^{\prime \prime} t}{q^{\prime \prime}}-\frac{t}{q^{\prime} q^{\prime \prime}}\right)=\varrho\left(\frac{p^{\prime \prime} t}{q^{\prime \prime}}\right)+\frac{t}{q^{\prime} q^{\prime \prime}} ;
$$


if $t$ is a multiple of $q^{\prime \prime}$ in (14), then we must subtract one from the right hand side.

By (1) we have

$$
\begin{aligned}
D\left(\frac{p}{q}\right) & =\sum_{t=1}^{q-1}\left(\frac{1}{2}-\frac{t}{q}\right) \varrho\left(\frac{p t}{q}\right)=-\frac{1}{q} \sum_{t=1}^{q-1} t \varrho\left(\frac{p t}{q}\right) \\
& =-\frac{1}{q} \sum_{t=1}^{q-1} t \varrho\left(\frac{p^{\prime} t}{q^{\prime}}\right)+\frac{1}{q^{2} q^{\prime}} \sum_{t=1}^{q-1} t^{2} \\
& =-\frac{1}{q} \sum_{t=1}^{a q^{\prime}+q^{\prime \prime}-1} t \varrho\left(\frac{p^{\prime} t}{q^{\prime}}\right)+\frac{(q-1)(2 q-1)}{6 q q^{\prime}} .
\end{aligned}
$$

In the first term in (15) we write $t=u q^{\prime}+v$ with $0 \leq u \leq a-1,1 \leq v \leq q^{\prime}$. The first term becomes

$$
-\frac{1}{q} \sum_{u=0}^{a-1} \sum_{v=1}^{q^{\prime}}\left(u q^{\prime}+v\right) \varrho\left(\frac{p^{\prime} v}{q^{\prime}}\right)-\frac{1}{q} \sum_{v=1}^{q^{\prime \prime}-1}\left(a q^{\prime}+v\right) \varrho\left(\frac{p^{\prime} v}{q^{\prime}}\right) .
$$

The terms with $v=q^{\prime}$ in (16) give

$$
-\frac{1}{q} \sum_{u=0}^{a-1}\left(u q^{\prime}+q^{\prime}\right) \varrho(0)=-\frac{a(a+1) q^{\prime}}{4 q}=\frac{(a+1)\left(q^{\prime \prime}-q\right)}{4 q} .
$$

The other terms in the first sum of (16) give

$$
\begin{aligned}
-\frac{1}{q} \sum_{u=0}^{a-1} \sum_{v=1}^{q^{\prime}-1}\left(u q^{\prime}+\frac{q^{\prime}}{2}\right) \varrho\left(\frac{p^{\prime} v}{q^{\prime}}\right)+\frac{a}{q} \sum_{v=1}^{q^{\prime}-1}\left(\frac{q^{\prime}}{2}-v\right) \varrho\left(\frac{p^{\prime} v}{q^{\prime}}\right) \\
=0+\frac{a q^{\prime}}{q} D\left(\frac{p^{\prime}}{q^{\prime}}\right)=\left(1-\frac{q^{\prime \prime}}{q}\right) D\left(\frac{p^{\prime}}{q^{\prime}}\right) .
\end{aligned}
$$

For the second term in (16) we use (14) instead of (13) to obtain

$$
\begin{aligned}
-\frac{1}{q} \sum_{v=1}^{q^{\prime \prime}-1}\left(a q^{\prime}+v\right) \varrho\left(\frac{p^{\prime \prime} v}{q^{\prime \prime}}\right)-\frac{1}{q} \sum_{v=1}^{q^{\prime \prime}-1} \frac{\left(a q^{\prime}+v\right) v}{q^{\prime} q^{\prime \prime}} \\
=\frac{q^{\prime \prime}}{q} D\left(\frac{p^{\prime \prime}}{q^{\prime \prime}}\right)-\frac{a\left(q^{\prime \prime}-1\right)}{2 q}-\frac{\left(q^{\prime \prime}-1\right)\left(2 q^{\prime \prime}-1\right)}{6 q^{\prime} q^{\prime \prime}},
\end{aligned}
$$

as in (17). Collecting terms together, we obtain (12), the result of the lemma.

The three-term recurrence is in second difference form, so we obtain the theorem after two inductions. The result of the first induction is a form of the reciprocity formula (7), noted by Rademacher [8]. Consequently, Lemma 2 gives an independent proof of the reciprocity formula. 
Lemma 2. If $p^{\prime} / q^{\prime}$ and $p / q$ are consecutive convergents of a continued fraction, with

$$
\frac{p}{q}-\frac{p^{\prime}}{q^{\prime}}= \pm \frac{1}{q q^{\prime}},
$$

then

$$
D\left(\frac{p}{q}\right)-D\left(\frac{p^{\prime}}{q^{\prime}}\right)= \pm\left(\frac{q^{2}+q^{\prime 2}+1}{12 q q^{\prime}}-\frac{1}{4}\right),
$$

the \pm sign agreeing with (18).

COROLlaRY (reciprocity formula).

$$
D\left(\frac{q^{\prime}}{q}\right)+D\left(\frac{q}{q^{\prime}}\right)=\frac{q^{2}+q^{\prime 2}+1}{12 q q^{\prime}}-\frac{1}{4} .
$$

Proof of Lemma 2. To begin the induction, we note that

$$
\begin{gathered}
D\left(p_{0} / q_{0}\right)=D\left(a_{0}\right)=0 \\
D\left(p_{1} / q_{1}\right)=D\left(a_{0}+1 / a_{1}\right)=D\left(1 / a_{1}\right)=\sum_{t=1}^{q_{1}-1}\left(\frac{1}{2}-\frac{t}{q_{1}}\right)=\frac{q_{1}^{2}+2}{12 q_{1}}-\frac{1}{4},
\end{gathered}
$$

in the notation introduced above for continued fractions. This verifies (19) when $p / q$ is $p_{1} / q_{1}, p^{\prime} / q^{\prime}$ is $p_{0} / q_{0}$.

We treat the induction step in the case when the sign in (18) is + . In the notation of Lemma 1, we take as the induction hypothesis that

$$
D\left(\frac{p^{\prime}}{q^{\prime}}\right)-D\left(\frac{p^{\prime \prime}}{q^{\prime \prime}}\right)=-\frac{q^{2}+q^{\prime \prime 2}+1}{12 q^{\prime} q^{\prime \prime}}+\frac{1}{4} .
$$

By Lemma 1 we have

$$
\begin{aligned}
D\left(\frac{p}{q}\right) & -D\left(\frac{p^{\prime}}{q^{\prime}}\right) \\
& =-\frac{q^{\prime \prime}}{q}\left(D\left(\frac{p^{\prime}}{q^{\prime}}\right)-D\left(\frac{p^{\prime \prime}}{q^{\prime \prime}}\right)\right)+\frac{a}{12}\left(1+\frac{q^{\prime \prime}}{q}\right)-\frac{1}{4}\left(1-\frac{q^{\prime \prime}}{q}\right) \\
& =\frac{q^{\prime 2}+q^{\prime \prime 2}+1}{12 q q^{\prime}}-\frac{1}{4}+\frac{a}{12} \frac{\left(q+q^{\prime \prime}\right)}{q},
\end{aligned}
$$

and the result follows since

$$
\frac{a}{12} \frac{\left(q+q^{\prime \prime}\right)}{q}=\frac{\left(q-q^{\prime \prime}\right)\left(q+q^{\prime \prime}\right)}{12 q q^{\prime}} .
$$

When the sign in (18) is reversed, then the signs in Lemma 1 and in (20) are both reversed. 
Proof of the Corollary. Again there are two cases. When the sign in $(18)$ is + , then $p q^{\prime} \equiv 1(\bmod q)$, so that by $(5)$

$$
D(p / q)=D\left(q^{\prime} / q\right) \text {. }
$$

However, $p^{\prime} q \equiv-1\left(\bmod q^{\prime}\right)$, so that

$$
D\left(\frac{q}{q^{\prime}}\right)=D\left(\frac{\left(a_{0}+1\right) q^{\prime}-p^{\prime}}{q^{\prime}}\right)=-D\left(\frac{p^{\prime}}{q^{\prime}}\right)
$$

by (5) and (6). When the sign in (18) is - , then the signs in (19), (21) and (22) are all reversed.

Proof of Theorem 1. Since $q_{0}=1, q_{1}=a_{1}$, we interpret $q_{-1}$ as 0 in the relation

$$
q_{1}=a_{1} q_{0}+q_{-1} .
$$

We then have

$$
\frac{p_{0}+q_{-1}}{q_{0}}-a_{0}=0=D\left(\frac{p_{0}}{q_{0}}\right) .
$$

For the induction step we put $p_{i} / q_{i}=p / q$, and use the notation $q=a q^{\prime}+q^{\prime \prime}$ of Lemma 1 with $a=a_{i}$ etc. As usual there are two cases. If $i$ is odd, then we take the + sign in (19) to get

$$
\begin{aligned}
12 D\left(\frac{p}{q}\right)-12 D\left(\frac{p^{\prime}}{q^{\prime}}\right) & =\frac{q}{q^{\prime}}+\frac{q^{\prime}}{q}-3+\frac{p}{q}-\frac{p^{\prime}}{q^{\prime}} \\
& =\frac{p+q^{\prime}}{q}-\frac{p^{\prime}-q^{\prime \prime}}{q^{\prime}}+a-3 .
\end{aligned}
$$

If $i$ is even, then we take the - sign in (19) to get

$$
12 D\left(\frac{p}{q}\right)-12 D\left(\frac{p^{\prime}}{q^{\prime}}\right)=\frac{p-q^{\prime}}{q}-\frac{p^{\prime}+q^{\prime \prime}}{q^{\prime}}-a+3 .
$$

The theorem now follows by induction, using (23) and (24) for alternate values of $i$ up to $i=n$.

Many properties of Dedekind sums may be deduced at once from the theorem. The following results are due to Rademacher [8]. If $12 D(p / q)$ is an integer, then

$$
\begin{gathered}
q_{n-1} \equiv(-1)^{n} p_{n}\left(\bmod q_{n}\right), \\
D\left(q_{n-1} / q_{n}\right)=(-1)^{n} D\left(p_{n} / q_{n}\right)
\end{gathered}
$$

by (6). However, from (9)

$$
p_{n} q_{n-1} \equiv(-1)^{n-1}\left(\bmod q_{n}\right),
$$

so that by (5) and (6)

$$
D\left(q_{n-1} / q_{n}\right)=(-1)^{n-1} D\left(p_{n} / q_{n}\right) .
$$


We deduce that $D(p / q)$ must be zero. In particular, $D(p / q)$ never takes the values $1,2,3, \ldots$

The first use of Dedekind sums [4] was to compute the multiplier system for the Dedekind eta function. Let $M=\left(\begin{array}{ll}a & b \\ c & d\end{array}\right)$ be a matrix with positive integer entries and determinant one. We note that $a / c$ and $b / d$ must be consecutive convergents for some continued fraction (if we allow the last partial quotient to be one), and similarly for the other ratios. There are eight associated Dedekind sums, in four pairs:

$$
\begin{array}{llrl}
D(a / c)=D(d / c), & & D(a / b)=D(d / b), \\
D(c / a)=-D(b / a), & & D(c / d)=-D(b / d) .
\end{array}
$$

We have

$$
\begin{aligned}
& D\left(\frac{a}{c}\right)-D\left(\frac{b}{d}\right)=\frac{c^{2}+d^{2}+1}{12 c d}-\frac{1}{4}=\frac{a+d}{12 c}-\frac{b-c}{12 d}-\frac{1}{4}, \\
& D\left(\frac{a}{b}\right)-D\left(\frac{c}{d}\right)=\frac{b^{2}+d^{2}+1}{12 b d}-\frac{1}{4}=\frac{a+d}{12 b}-\frac{c-b}{12 d}-\frac{1}{4}, \\
& D\left(\frac{d}{c}\right)-D\left(\frac{b}{a}\right)=\frac{a^{2}+c^{2}+1}{12 a c}-\frac{1}{4}=\frac{a+d}{12 c}-\frac{b-c}{12 a}-\frac{1}{4}, \\
& D\left(\frac{d}{b}\right)-D\left(\frac{c}{a}\right)=\frac{a^{2}+b^{2}+1}{12 a b}-\frac{1}{4}=\frac{a+d}{12 b}-\frac{c-b}{12 a}-\frac{1}{4} .
\end{aligned}
$$

We deduce that the various Dedekind sums are related by

$$
\begin{aligned}
-D\left(\frac{a}{c}\right)+\frac{a+d}{12 c}-\frac{1}{4} & =D\left(\frac{d}{b}\right)-\frac{a+d}{12 b}+\frac{1}{4} \\
& =D\left(\frac{c}{d}\right)-\frac{c-b}{12 d}=D\left(\frac{c}{a}\right)-\frac{c-b}{12 a}=\frac{1}{12} \psi(M),
\end{aligned}
$$

where $\psi(M)$ is an integer that determines the twenty-fourth root of unity in the formula for $\eta(M \tau)$. For two such matrices $M$ and $N$, there should be a relation between $\psi(M), \psi(N)$ and $\psi(M N)$.

There is a continued fraction with $a / c$ and $b / d$ as a consecutive convergents:

and if $c \geq d>0$, then

$$
\frac{a}{c}=a_{0}+\frac{1}{a_{1}+} \ldots \frac{1}{a_{t}}
$$

$$
\begin{gathered}
\frac{b}{d}=a_{0}+\frac{1}{a_{1}+} \ldots \frac{1}{a_{t-1}}, \\
D\left(\frac{a}{c}\right)=\frac{1}{12}\left(\frac{p_{t}+q_{t-1}}{q_{t}}-a_{0}+a_{1}-\ldots+a_{t}\right)-\frac{1}{4},
\end{gathered}
$$


but if $d>c>0$, then

$$
\frac{b}{d}=a_{0}+\frac{1}{a_{1}+} \ldots \frac{1}{a_{t+1}},
$$

where $a_{t+1}$ is the largest integer strictly less than $d / c$, and

$$
\begin{aligned}
D\left(\frac{a}{c}\right) & =\frac{1}{12}\left(\frac{p_{t}+q_{t-1}}{q_{t}}-a_{0}+a_{1}-\ldots+a_{t}\right)-\frac{1}{4} \\
& =\frac{1}{12}\left(\frac{p_{t}+q_{t+1}}{q_{t}}-a_{0}+a_{1}-\ldots+a_{t}-a_{t+1}\right)-\frac{1}{4} .
\end{aligned}
$$

Here we may have $a_{t}=1$ or $a_{t+1}=1$. If we define $a_{t+1}$ in both cases to be the greatest integer strictly less than $d / c$, which is zero when $c \geq d$, then in both cases

$$
D\left(\frac{a}{c}\right)=\frac{1}{12}\left(\frac{a+d}{c}-a_{0}+a_{1}-\ldots+a_{t}-a_{t+1}\right)-\frac{1}{4} .
$$

Similarly, if $N=\left(\begin{array}{ll}f & e \\ s & r\end{array}\right)$ is another integer matrix with

$$
\frac{f}{s}=f_{0}+\frac{1}{f_{1}+} \cdots \frac{1}{f_{u}},
$$

and $f_{u+1}$ is the greatest integer strictly less than $r / s$, then

$$
D\left(\frac{f}{s}\right)=\frac{1}{12}\left(\frac{f+r}{s}-f_{0}+f_{1}-\ldots+f_{u}-f_{u+1}\right)-\frac{1}{4} .
$$

We have

$$
M N=\left(\begin{array}{ll}
a f+b s & a e+b r \\
c f+d s & c e+d r
\end{array}\right)
$$

and

$$
\frac{r}{s}-\frac{c e+d r}{c f+d s}=\frac{c}{s(c f+d s)}<\frac{1}{s} .
$$

Since any integer strictly less than $r / s$ is at most $(r-1) / s$, we see that $f_{u+1}$ is also the greatest integer strictly less than $(c e+d r) /(c f+d s)$. Now we form the continued fraction for $(c e+d r) /(c f+d s)$. We note the zero drop-out rule, that

$$
b_{0}+\frac{1}{b_{1}+} \cdots \frac{1}{b_{v}+} \frac{1}{0+} \frac{1}{b_{v+2}+} \cdots \frac{1}{b_{v+w}}=b_{0}+\frac{1}{b_{1}+} \cdots \frac{1}{b_{v}+b_{v+2}+} \cdots \frac{1}{b_{v+w}},
$$

the alternating sum of the $b_{i}$ remaining unchanged. There are two cases: if $c \geq d>0$, then we write

$$
\frac{a f+b s}{c f+d s}=\frac{p_{t} f / s+p_{t-1}}{q_{t} f / s+q_{t-1}}=a_{0}+\frac{1}{a_{1}+} \ldots \frac{1}{a_{t}+} \frac{1}{f_{0}+} \ldots \frac{1}{f_{u}},
$$


by the rules for continued fractions. If $d>c>0$, then

$$
\begin{aligned}
\frac{a f+b s}{c f+d s} & =\frac{p_{t}+p_{t+1} s / f}{q_{t}+q_{t+1} s / f}=a_{0}+\frac{1}{a_{1}+} \cdots \frac{1}{a_{t+1}+} \frac{1}{0+} \frac{1}{f_{0}+} \cdots \frac{1}{f_{u}} \\
& =a_{0}+\frac{1}{a_{1}+} \cdots \frac{1}{a_{t+1}+f_{0}+} \cdots \frac{1}{f_{u}} .
\end{aligned}
$$

Whether or not we have to invoke the zero drop-out rule again, we have

(25) $\psi(M N)=a_{0}-a_{1}+\ldots-a_{t}+a_{t+1}+f_{0}-\ldots-f_{u}+f_{u+1}=\psi(M)+\psi(N)$.

When we put

$$
\psi\left(\begin{array}{ll}
1 & b \\
0 & 1
\end{array}\right)=b, \quad \psi\left(\begin{array}{ll}
1 & 0 \\
c & 1
\end{array}\right)=-c,
$$

then $\psi$ is defined on the semigroup of integer matrices with non-negative entries, and gives a homomorphism into $\mathbb{Z}$, the additive group of integers. The semigroup is generated by

$$
R=\left(\begin{array}{ll}
1 & 0 \\
1 & 1
\end{array}\right), \quad S=\left(\begin{array}{ll}
1 & 1 \\
0 & 1
\end{array}\right) .
$$

Rademacher [8] gives a rule for defining $\psi(M)$ for all matrices of the group $\mathrm{SL}(2, \mathbb{Z})$. The identity $(25)$ must be replaced by a congruence, since the group contains a matrix $T=\left(\begin{array}{cc}0 & 1 \\ -1 & 0\end{array}\right)$ with

$$
T^{2}=-I, \quad(S T)^{3}=I, \quad R=T^{-1} S^{-1} T .
$$

The identity (25) can be written as

$$
D\left(\frac{a}{c}\right)+D\left(\frac{f}{s}\right)-D\left(\frac{a f+b s}{c f+d s}\right)=\frac{c^{2}+s^{2}+(c f+d s)^{2}}{12 c s(c f+d s)}-\frac{1}{4},
$$

analogous to the functional equation (8). We note that if $p+q+r+s=0$, then

$$
\left(\begin{array}{cc}
p & -s \\
r & q
\end{array}\right)\left(\begin{array}{cc}
q & -s \\
p & r
\end{array}\right)\left(\begin{array}{cc}
r & -s \\
q & p
\end{array}\right)=-(p+q)(p+r)(q+r) I .
$$

The matrices on the left have determinants greater than one. The matrix identity (27) suggests that (8) and (26) may be special cases of some more complicated identity.

When we translate (26) into the notation of continued fractions, then we find a new identity for Dedekind sums.

THEOREM 2 (interpolation formula). For $x$ rational we write

$$
E(x)=D(x)-x / 6 .
$$


Suppose that $p / q, p^{\prime} / q^{\prime}$ and $p^{\prime \prime} / q^{\prime \prime}$ are rational numbers in their lowest terms with

$$
\frac{p}{q}=\frac{u p^{\prime}+v p^{\prime \prime}}{u q^{\prime}+v q^{\prime \prime}}, \quad \frac{p^{\prime}}{q^{\prime}}-\frac{p^{\prime \prime}}{q^{\prime \prime}}=\frac{1}{q^{\prime} q^{\prime \prime}},
$$

where $u$ and $v$ are positive integers with $(u, v)=1$. Then

$$
E\left(\frac{p}{q}\right)=\lambda\left(E\left(\frac{p^{\prime}}{q^{\prime}}\right)-E\left(\frac{v}{u}\right)\right)+(1-\lambda)\left(E\left(\frac{p^{\prime \prime}}{q^{\prime \prime}}\right)+E\left(\frac{u}{v}\right)\right),
$$

where $\lambda=u q^{\prime} /\left(u q^{\prime}+v q^{\prime \prime}\right)$ is defined by

$$
\frac{p}{q}=\lambda \frac{p^{\prime}}{q^{\prime}}+(1-\lambda) \frac{p^{\prime \prime}}{q^{\prime \prime}} .
$$

Proof. We use the notation of (26), writing $a / c, b / d$ and $f / s$ for $p^{\prime} / q^{\prime}$, $p^{\prime \prime} / q^{\prime \prime}$ and $u / v$, so that

$$
\lambda=\frac{c f}{c f+d s}, \quad 1-\lambda=\frac{d s}{c f+d s} .
$$

We write $(26)$ as

$$
D\left(\frac{a f+b s}{c f+d s}\right)=D\left(\frac{a}{c}\right)+D\left(\frac{f}{s}\right)-\frac{c^{2}+s^{2}+(c f+d s)^{2}}{12 c s(c f+d s)}+\frac{1}{4} .
$$

By Lemma 2 applied to $a / c$ and $b / d$, we have

$$
D\left(\frac{a}{c}\right)=\lambda D\left(\frac{a}{c}\right)+(1-\lambda) D\left(\frac{b}{d}\right)-\frac{s^{2}\left(c^{2}+d^{2}+1\right)}{12 c s(c f+d s)}-\frac{1-\lambda}{4},
$$

so that

$$
\begin{aligned}
D\left(\frac{a f+b s}{c f+d s}\right)= & \lambda D\left(\frac{a}{c}\right)+(1-\lambda) D\left(\frac{b}{d}\right)+D\left(\frac{f}{s}\right)+\frac{\lambda}{4} \\
& +\frac{c^{2} s^{2}-c^{2} f^{2}-c^{2}-2 c d f s}{12 c s(c f+d s)}
\end{aligned}
$$

By the Corollary to Lemma 2 for $f / s$, we have

$$
\frac{\lambda}{4}=\frac{c^{2}\left(f^{2}+s^{2}+1\right)}{12 c s(c f+d s)}-\lambda D\left(\frac{f}{s}\right)-\lambda D\left(\frac{s}{f}\right)
$$


and

$$
\begin{aligned}
D\left(\frac{a f+b s}{c f+d s}\right)= & \lambda D\left(\frac{a}{c}\right)+(1-\lambda) D\left(\frac{b}{d}\right)-\lambda D\left(\frac{s}{f}\right)+(1-\lambda) D\left(\frac{f}{s}\right) \\
& +\frac{2 c s-2 d f}{12(c f+d s)} \\
= & \lambda\left(D\left(\frac{a}{c}\right)-D\left(\frac{s}{f}\right)+\frac{s}{6 f}\right) \\
& +(1-\lambda)\left(D\left(\frac{b}{d}\right)+D\left(\frac{f}{s}\right)-\frac{f}{6 s}\right),
\end{aligned}
$$

which gives the result when we substitute for $D(x)$ in terms of $E(x)$.

Rademacher [8] left many open questions about the value distribution of Dedekind sums. Hickerson [5] settled the conjecture that the values of the inhomogeneous sum $D(p / q)$ are dense on the real line, by showing that the points $(p / q, D(p / q))$ are dense in $\mathbb{R}^{2}$. The construction again uses continued fractions. Given $\alpha$ and $\beta$ real and $\varepsilon>0$, we can specify $a_{0}, \ldots, a_{r}$ so that all continued fractions beginning

$$
a_{0}+\frac{1}{a_{1}+} \ldots \frac{1}{a_{r}+}
$$

lie in the interval $(\alpha-\varepsilon, \alpha+\varepsilon)$, and $b_{1}, \ldots, b_{s}$ so that all continued fractions beginning

$$
b_{1}+\frac{1}{b_{2}+} \ldots \frac{1}{b_{s}+}
$$

lie in an interval $(\gamma-\varepsilon, \gamma+\varepsilon)$, where

$$
(\alpha+\gamma) / 12-\beta=e,
$$

an integer. Here $a_{0}$ is an integer, $a_{1}, \ldots, a_{r}, b_{1}, \ldots, b_{s}$ are positive integers. We choose an odd integer $n \geq r+s+2$. We put $a_{n}=b_{1}, a_{n-1}=$ $b_{2}, \ldots, a_{n-s+1}=b_{1}$, and choose $a_{r+1}, \ldots, a_{n-s}$ so that

$$
a_{0}-a_{1}+\ldots-a_{n}=e-3 .
$$

Then

$$
\frac{p}{q}=\frac{p_{n}}{q_{n}}=a_{0}+\frac{1}{a_{1}+} \cdots \frac{1}{a_{n}}
$$

lies in the interval $(\alpha-\varepsilon, \alpha+\varepsilon)$, with $q_{n-1} / q_{n}$ in the interval $(\gamma-\varepsilon, \gamma+\varepsilon)$, and $D(p / q)$ in the interval $(\beta-\varepsilon / 6, \beta+\varepsilon / 6)$ as required.

The points $(p / q, D(p / q))$ in two dimensions are concentrated close to the $x$-axis. The uniform distribution results of Vardi [9] and Myerson [7] use Weyl's criterion and bounds for generalized Kloosterman sums. The classical Kloosterman sum bound corresponds to the discrepancy of the sequence in the unit square for which $x_{n}$ runs through the rational numbers 
$p / q$ (ordered lexicographically in their lowest terms form), and $y_{n}$ through the numbers $12 D(p / q)$, reduced modulo one. The discrepancy of the first $N$ terms of this sequence is easily seen to be $O\left(N^{3 / 4} \log ^{3} N\right)$; this estimate could be improved using Kuznetsov's work on sums of Kloosterman sums. Bruggeman $[2,3]$ has investigated the points $(p / q, D(p / q) / q)$ in $\mathbb{R}^{2}$, using the representation theory of the group $\mathrm{SL}(2, \mathbb{Z})$. It would be of interest to investigate the distribution of Dedekind sums using the metrical theory of continued fractions.

\section{References}

[1] P. Barkan, Sur les sommes de Dedekind et les fractions continues finies, C. R. Acad. Sci. Paris Sér. A 284 (1977), 923-926.

[2] R. W. Bruggeman, On the distribution of Dedekind sums, in: Automorphic Functions and their Applications, U.S.S.R. Acad. Sci., Khabarovsk 1990, 82-89.

[3] - Eisenstein series and the distribution of Dedekind sums, to appear.

[4] R. Dedekind, Erläuterung zu den Fragmenten xxviii, in: Bernhard Riemanns gesammelte mathematische Werke, Dover, New York 1953.

[5] D. Hickerson, Continued fractions and density results for Dedekind sums, J. Reine Angew. Math. 290 (1977), 113-116.

[6] D. E. Knuth, Notes on generalized Dedekind sums, Acta Arith. 33 (1977), 297-325.

[7] G. Myerson, Dedekind sums and uniform distribution, J. Number Theory 28 (1988), 233-239.

[8] H. Rademacher, Dedekind Sums (E. Grosswald, ed.), Carus Math. Monographs, 1972 .

[9] I. Vardi, A relation between Dedekind sums and Kloosterman sums, Duke Math. J. 55 (1987), 189-197.

DEPARTMENT OF MATHEMATICS

UNIVERSITY OF YORK

YORK YO1 5DD, ENGLAND
SCHOOL OF MATHEMATICS UNIVERSITY OF WALES COLLEGE OF CARDIFF CARDIFF CF2 4AG, WALES 